\title{
A pesquisa científica sob o olhar dos acadêmicos de enfermagem
}

\author{
Scientific research under the look of nursing academics
}

Investigación científica bajo la mirada de las academias de enfermería

\begin{abstract}
Larisse Batista Wanzeler ${ }^{1 *}$, Amanda Carolina Rozario Pantoja ${ }^{1}$, Danilo Sousa das Mercês ${ }^{1}$, Bruno de Jesus Castro dos Santos ${ }^{1}$, Maicon de Araujo Nogueira ${ }^{1}$, Thalyta Mariany Rêgo Lopes Ueno ${ }^{2}$, Renata Glaucia Barros da Silva Lopes ${ }^{3}$, Marta Solange Camarinha Ramos Costa ${ }^{4}$, Cintia Yolette Urbano Pauxis Aben-Athar Valentim ${ }^{4}$, Aline Maria Pereira Cruz Ramos ${ }^{4}$
\end{abstract}

\section{RESUMO}

Objetivo: Identificar a percepção do graduando de enfermagem sobre a participação na pesquisa científica durante a graduação. Métodos: Estudo multicêntrico, descritivo, exploratório com abordagem quantiqualitativa, realizado com acadêmicos de enfermagem de duas instituições de ensino superior privada de Belém, Estado do Pará, Brasil. O estudo ocorreu entre fevereiro a março de 2019, aplicou-se um questionário para 102 alunos do curso de enfermagem sendo estudo aprovado pelos comitês de ética em pesquisa. Resultados: Média de idade foi de 31,09 ( $\pm 6,23)$, predominando o gênero feminino 86,3\%, matriculados no turno matutino $53,9 \%$, que experimentam uma lacuna entre o estímulo e a produção científica no contexto de formação acadêmica. Salientou-se pelos participantes, as dificuldades na elaboração do Trabalho de Conclusão de Curso (TCC), insatisfação com a disciplina metodologia científica e pouco interesse pela pesquisa após formação. Conclusão: Evidenciam-se dificuldades na elaboração do trabalho de conclusão de curso, a insatisfação com a disciplina metodologia científica e pouco interesse pela pesquisa após formação. A experiência negativa vivenciada desfocou a pesquisa científica como principal opção de escolha profissional futura.

Palavras-chave: Estudantes de enfermagem, Metodologia, Pesquisa em enfermagem, Educação em enfermagem, Aprendizagem.

\section{ABSTRACT}

Objective: To demonstrate the perception of nursing undergraduates about their participation in scientific research during undergraduate studies. Methods: A multicenter, descriptive, exploratory study with a quantitative and qualitative approach, conducted with nursing students from two private higher education institutions in Belém, State of Pará, Brazil. The study took place between February and March 2019, and a questionnaire was applied to 102 students of the nursing course. This study was approved by the research ethics committees. Results: The average age was $31.09 \pm(6.23)$, predominantly female $86.3 \%$, enrolled in the morning shift $53.9 \%$, who experience a gap between stimulus and scientific production in the context of academic education. The participants highlighted difficulties in the elaboration of the Course Conclusion Paper (TCC), dissatisfaction with the scientific methodology discipline and little interest in research after graduation. Conclusion: It was evidenced difficulties in the elaboration of the course conclusion work, dissatisfaction with

\footnotetext{
${ }^{1}$ Universidade da Amazônia (UNAMA), Belém - Pará. *E-mail: larissewanzeler@gmail.com

2 Universidade do Estado do Pará (UEPA), Belém - Pará.

${ }^{3}$ Hospital Universitário João de Barros Barreto (HUJBB), Belém - Pará.

${ }^{4}$ Faculdade de Enfermagem, Universidade Federal do Pará (UFPA), Belém - Pará.
}

SUBMETIDO EM: 11/2019

ACEITO EM: 12/2019

PUBLICADO EM: 2/2020

REAS/EJCH | Vol.12(2) | e2503 | DOI: https://doi.org/10.25248/reas.e2503.2020 Página 1 de 10 
the scientific methodology discipline and little interest in research after training. The negative experience lived defocused scientific research as the main option for future professional choice.

Keywords: Nursing students, Methodology, Nursing research, Nursing education, Learning.

\section{RESUMEN}

Objetivo: Demostrar la percepción de los estudiantes de pregrado de enfermería sobre su participación en la investigación científica durante los estudios de pregrado. Métodos: Estudio exploratorio, multicéntrico, descriptivo, con enfoque cuantitativo y cualitativo, realizado con estudiantes de enfermería de dos instituciones privadas de educación superior en Belém, Estado de Pará, Brasil. El estudio se realizó entre febrero y marzo de 2019, y se aplicó un cuestionario a 102 estudiantes del curso de enfermería, que fue aprobado por los comités de ética de investigación. Resultados: La edad promedio fue de $31.09 \pm(6.23)$, predominantemente mujeres $86.3 \%$, inscritas en el turno matutino $53.9 \%$, que experimentan una brecha entre el estímulo y la producción científica en el contexto de la educación académica. Los participantes destacaron las dificultades para preparar el Documento de finalización del curso (CCT), la insatisfacción con la disciplina de la metodología científica y el poco interés en la investigación después de la capacitación. Conclusión: Se evidenciaron dificultades en la elaboración del trabajo de conclusión del curso, insatisfacción con la disciplina de la metodología científica y poco interés en la investigación después de la capacitación. La experiencia negativa vivió la investigación científica desenfocada como la principal opción para la futura elección profesional.

Palabras-clave: Estudiantes de enfermería, Metodología, Investigación en enfermería, Educación en enfermería, Aprendizaje.

\section{INTRODUÇÃO}

A Resolução no 573/2018 institui as Diretrizes Curriculares Nacionais do Curso de Graduação em Enfermagem, nela ficou estabelecido que a formação do enfermeiro deve ser pautada na visão crítica, associada às Práticas Baseadas em Evidências (PBE), permitindo, por conseguinte, a valorização da prática profissional (SILVA IR, et al., 2017).

A inserção da pesquisa na graduação é a oportunidade de ressignificar a formação do profissional, antes focada apenas na visão assistencial e, agora, busca formar profissionais mais completos e dinâmicos, denominados como pesquisador-assistencial ou enfermeiro cientista. Processo também conhecido como PBE, capaz de implementar intervenções consolidadas cientificamente. Alternativamente, dessa prática assistencial podem-se identificar problemas que gerem hipóteses passíveis de pesquisa e resolubilidade (CAMARGO FC, et al., 2018).

É a pesquisa que impulsiona a construção do conhecimento e pensamento crítico. Para tal, o estímulo à produção cientifica, produto oriundo da pesquisa, deve ocorrer ainda na graduação sendo o docente 0 mediador durante todo o processo (MORAES A, et al., 2018). A participação do acadêmico em grupos de pesquisa é uma valiosa oportunidade de desenvolver as habilidades da metodologia cientifica. Essa aproximação facilita a vivência da pesquisa e produção do conhecimento científico, estabelece vínculos entre acadêmicos e pesquisadores e, ainda, resulta em produção cientifica (ARAÚJO AML, et al., 2015).

No entanto, o cenário brasileiro não consegue suprir a demanda acadêmica devido a alguns entraves como a existência de poucos grupos de pesquisa em enfermagem, com maior concentração na região Sudeste, reduzido número de doutores e limitado estímulo político-financeiro e a escassez ainda é maior nas instituições privadas (SCOCHI CGS, et al., 2015).

Outro entrave está relacionado ao acadêmico, cuja transição do ensino médio ao superior provoca mudanças e gera dificuldades significativas, grande parte delas relacionada à metodologia científica e sua aplicabilidade (PRAÇA FSG, 2015). Trata-se de uma disciplina obrigatória à diretriz curricular, que dita 
normatizações essenciais para aumentar a produção e potencializar a qualidade sua produção textual científica. Ela possibilita a produção de resumos, artigos e estrutura todo o Trabalho de Conclusão de Curso (TCC) que é o primeiro contato do acadêmico com a pesquisa na maioria dos casos (OLIVEIRA TAB e VALENÇA KFP, 2015).

Entretanto, esta disciplina tão relevante costuma ter uma abordagem tradicionalmente teórica e isso tende a impactar na afinidade e dificultada da escrita científica do acadêmico de enfermagem (ARAÚJO AML, et al., 2015). Diante disso, o presente estudo teve como objetivo identificar a percepção do acadêmico de enfermagem sobre a participação na pesquisa científica durante a graduação.

\section{MÉTODOS}

Trata-se de um estudo multicêntrico, descritivo, exploratório com abordagem quanti-qualitativa. $O$ estudo foi realizado em duas Instituições de Ensino Superior (IES) privadas de Belém, Estado do Pará, norte brasileiro. A amostra inicial da pesquisa foi composta por 225 participantes, tendo como critério de inclusão alunos do último semestre do curso de graduação de enfermagem nos turnos manhã, tarde e noite, no período de fevereiro a março de 2019.

Para a coleta de dados, utilizou-se um questionário elaborado pelos autores, contendo treze questões, destas, dez eram fechadas e três abertas. As últimas estavam relacionadas à motivação dos acadêmicos sobre os processos que permeiam a investigação/pesquisa científica na formação.

Os participantes da pesquisa foram identificados com as seguintes denominações "G1, G2" e "H1, H2", sucessivamente, onde "G" e "H" representam as instituições de ensino e os números a ordem em que foram abordados.

A abordagem dos participantes ocorreu de forma individual em sala de aula, objetivando promover o conforto e privacidade na participação no estudo, além de preservar o anonimato e confidencialidade das informações. O tempo de preenchimento do questionário durou em média quinze minutos. As informações foram tabuladas em uma planilha do Programa Microsoft Excel ${ }^{\circledR}$ versão 2013 e submetidos ao teste estatístico Qui-quadrado de Pearson por meio do Programa Winpepi® (ABRAMSON JH, 2011).

Válido ressaltar, que o presente estudo apresentou limitações significativas quanto a redução do tamanho amostral, pois o critério de inclusão abordado foi a realização da coleta com alunos do último semestre do curso de graduação de enfermagem. Partindo dessa premissa, alguns acadêmicos deste período estavam em estágio supervisionado obrigatório, enquanto outros em aula teórica, metodologia aplicada pela grade curricular das IES participantes, desta forma reduzindo a amostra inicial de 225 para 102 acadêmicos. Sendo assim, adotou-se como erro amostral uma margem de $5 \%$, p-valor < 0,05, com nível de confiança de $95 \%$, para a amostra final de 102 acadêmicos.

As respostas às perguntas abertas foram transcritas para o Programa Microsoft Word® versão 2013, e para a análise, utilizou-se o referencial metodológico proposto por Bardin (BARDIN L, 2011). A abordagem dos participantes ocorreu de forma individualizada e seguiu os pressupostos éticos da pesquisa com seres humanos, conforme as Resoluções no 466 de 2012 e 510 de 2016 do Conselho Nacional de Saúde (CNS) e Comissão Nacional de Ética em Pesquisa (CONEP). Este estudo foi aprovado pelos Comitês de Éticas e Pesquisas (CEP) das IES, sob o no 3.186.037 e 2.951. 095. Todos os participantes assinaram o Termo de Consentimento Livre e Esclarecido (TCLE).

\section{RESULTADOS}

\section{Caracterização dos acadêmicos de enfermagem}

A amostra do estudo foi constituída por 102 acadêmicos com média de idade de $31,09( \pm 6,23)$, a maioria era do gênero feminino $86,3 \%$ (88/102) e cursavam o período matutino 53,9\% (55/102) conforme a caracterização dos acadêmicos de enfermagem descritos (Tabela 1). 
Tabela 1 - Caracterização dos acadêmicos de enfermagem de duas instituições de ensino superior privadas, n=102. Belém, Pará, Brasil, 2019.

\begin{tabular}{cccc}
\hline Variáveis & $\mathbf{N}$ & $\%$ & $\boldsymbol{p}^{*}$ \\
\hline Idade & & & 0,934 \\
\hline $23-29$ & 48 & 47,1 & \\
$30-36$ & 36 & 35,3 & \\
$>40$ & 18 & 17,6 & \\
\hline Gênero & & & \\
\hline Feminino & 88 & 86,3 & \\
Masculino & 14 & 13,7 & \\
\hline Turno cursado & & & \\
\hline Matutino & 55 & 53,9 & \\
Vespertino & 19 & 18,6 & \\
Noturno & 28 & 27,5 & \\
\hline Total & 102 & $100 \%$ & \\
\hline
\end{tabular}

Legenda: *teste qui-quadrado de Pearson.

Fonte: Wanzeler LB, et al., 2019.

\section{Vivência da pesquisa na graduação}

Cerca de 69,6\% (71/102) dos acadêmicos afirmou ter sido estimulado a realizar pesquisa científica, embora a produção tenha sido limitada produção de resumo para congressos e eventos $62,7 \%(64 / 102)$. Evidenciou-se uma inexpressiva participação em projetos de pesquisa 13,7\% (14/102), publicação de livros 2,9 \% (3/102) ou artigos indexados 6,9 \% (7/102) dos participantes enquanto acadêmico (Tabela 2).

Tabela 2 - Vivências da pesquisa científica pelos acadêmicos de Enfermagem em duas instituições de ensino superior privadas, $\mathrm{n}=102$. Belém, Pará, Brasil, 2019.

\begin{tabular}{l|cccccccc}
\hline Variáveis & Sim & $\%$ & Não & $\%$ & NI & $\%$ & Total & $\boldsymbol{p}^{*}$ \\
\hline Participação em grupo de pesquisa & 14 & 13,7 & 87 & 85,3 & 1 & 1 & 102 & 0.380 \\
\hline Incentivo da instituição em pesquisa & 71 & 69,6 & 27 & 26,5 & 4 & 3,9 & 102 & 0.666 \\
\hline Trabalho apresentado em congresso & 64 & 62,7 & 37 & 36,3 & 1 & 1 & 102 & 0.712 \\
\hline Capítulo de livro publicado & 3 & 2,9 & 98 & 96,1 & 1 & 1 & 102 & 0.140 \\
\hline Artigo publicado em revista & 7 & 6,9 & 95 & 93,1 & - & - & 102 & 0.192 \\
\hline
\end{tabular}

Legenda: *teste qui-quadrado de Pearson; NI: não informado.

Fonte: Wanzeler LB, et al., 2019.

\section{A execução do trabalho de conclusão de curso}

Neste estudo, notou-se que a maioria dos acadêmicos iniciou a pesquisa científica no desenvolvimento do TCC, sendo que $48 \%$ (49/102) escolheu a sua temática durante o $7^{\circ}$ ao $9^{\circ}$ semestre, $64,7 \%$ (66/102) escolheu seu tema por decisão própria e $71,6 \%(73 / 102)$ afirmou estar satisfeito com a escolha. Adicionalmente, 0 motivo da escolha do orientador 47,1\% (48/102) foi justificada pela área de atuação do docente (Tabela 3 ). 
Tabela 3 - A execução do trabalho de conclusão de curso pelos acadêmicos de Enfermagem de duas instituições de ensino superior, em relação a elaboração do trabalho de conclusão de curso, $n=102$. Belém, Pará, Brasil, 2019.

\begin{tabular}{|c|c|c|c|}
\hline Variáveis & $\mathbf{N}$ & $\%$ & $p^{*}$ \\
\hline Semestre de escolha do tema & & & 0.864 \\
\hline $1^{\circ}-3^{\circ}$ semestre & 3 & 2,9 & \\
\hline $4^{\circ}-6^{\circ}=$ semestre & 24 & 23,5 & \\
\hline $7^{\circ}-9 \circ$ semestre & 49 & 48,0 & \\
\hline Não informado & 26 & 25,5 & \\
\hline Escolha do tema & & & 0.774 \\
\hline Própria & 66 & 64,7 & \\
\hline Orientador & 14 & 13,7 & \\
\hline Não informado & 22 & 21,6 & \\
\hline Satisfação com o tema escolhido & & & 0.659 \\
\hline Sim & 73 & 71,6 & \\
\hline Não & 8 & 7,8 & \\
\hline Não informado & 21 & 20,6 & \\
\hline $\begin{array}{l}\text { A escolha do orientador(a) foi baseada } \\
\text { em que aspecto? }\end{array}$ & & & 0.912 \\
\hline Na disciplina que ele ministra & 21 & 20,6 & \\
\hline $\mathrm{Na}$ escassez do tema & 17 & 16,7 & \\
\hline $\mathrm{Na}$ área de atuação do orientador & 48 & 47,1 & \\
\hline Não informado & 16 & 15,7 & \\
\hline Total & 102 & $100 \%$ & \\
\hline
\end{tabular}

Legenda: *teste qui-quadrado de Pearson.

Fonte: Wanzeler LB, et al., 2019.

\section{Expectativa de atuação profissional.}

Ao serem indagados sobre a área de atuação profissional após formados, 43,1\% (44/102) dos acadêmicos afirmou que pretende seguir a vertente assistencial, 28,4\% (29/102) na gestão, 18,6\% (19/102) na docência e uma minoria 9,8\% (10/102) optou pela pesquisa (Gráfico 1).

Gráfico 1 - Escolha da área de atuação profissional pelos graduandos de enfermagem de duas instituições de ensino superior, n=102. Belém, Pará, Brasil, 2019.

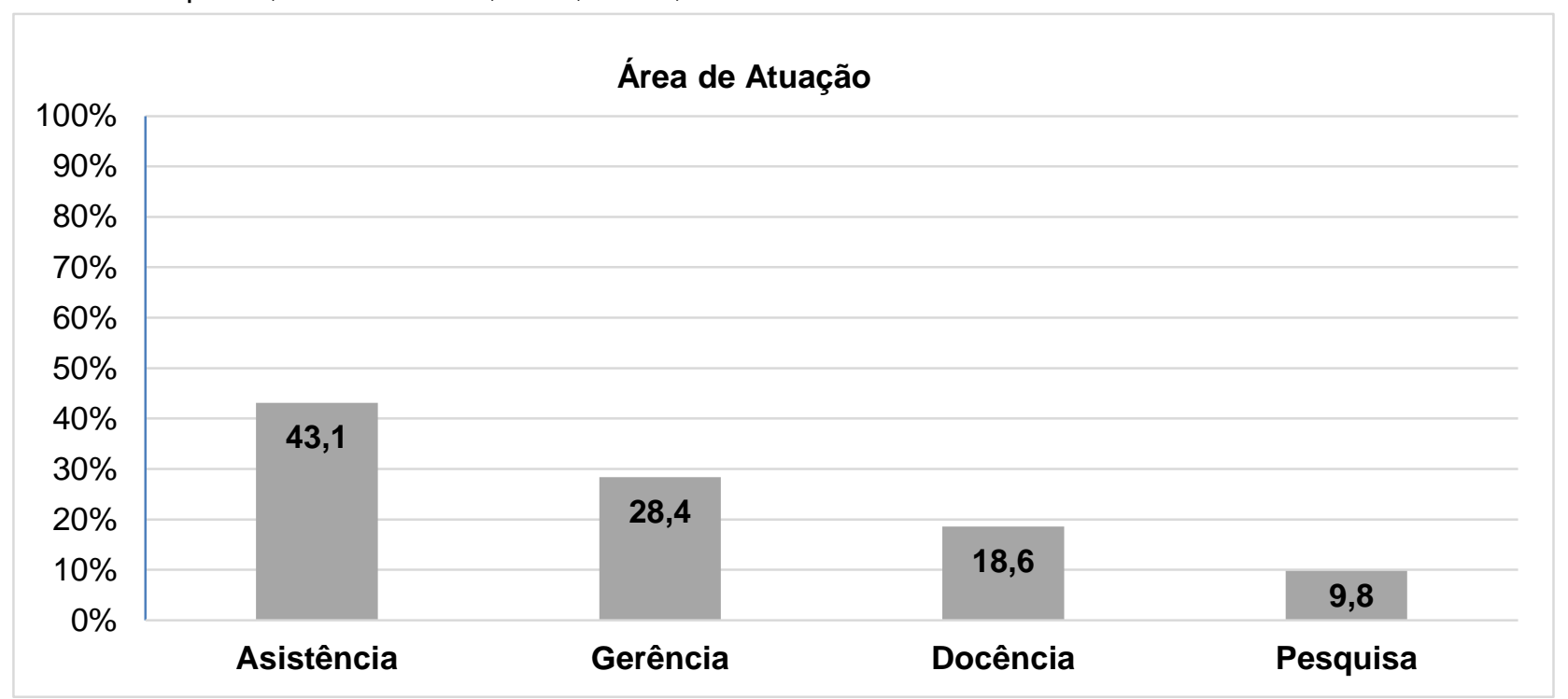

Legenda: *teste qui-quadrado de Pearson. p* 0,918

Fonte: Wanzeler LB, et al., 2019. 


\section{Relevância da pesquisa científica na percepção do acadêmico}

Nesta subcategoria, os participantes ressaltaram a importância da pesquisa científica na graduação, como ferramenta capaz de desenvolver habilidades assistenciais, pensamento crítico, bem como a melhoria do currículo lattes.

Isso eleva o conhecimento e também enriquece o currículo lattes preparando para as residências em enfermagem $(\mathrm{H} 07)$.

Enriquece o conhecimento do aluno e o faz ter uma visão crítica (H20).

Isso melhora a saúde e a assistência (G24).

\section{Percepção dos acadêmicos sobre a metodologia científica enquanto disciplina}

Dentre as IES's, uma apresentou a disciplina presencial e outra online (educação à distância - EAD) e por isso, notou-se divergência entre as falas dos acadêmicos. A maioria dos alunos da disciplina online relataram aversão à disciplina, dificuldade de aprendizado e pouca aplicabilidade. Alternativamente, a afinidade pela disciplina foi correlacionada ao professor que a ministrou presencialmente na outra IES.

Foi péssima! A nossa disciplina de metodologia científica foi em educação a distância, online, por conta disso temos muitas dificuldades (H02).

Aversão! É uma disciplina complexa e não houve um bom esclarecimento da mesma por ensino a distância (H09).

Afinidade por causa da metodologia do professor. (G11).

\section{Dificuldades dos acadêmicos na realização do trabalho de conclusão de curso}

Nesta categoria, a maioria apontou dificuldades de adequação às normas da Associação Brasileira de Normas Técnicas (ABNT), à construção da metodologia, dificuldades de inter-relação com o orientador, pesquisa sobre a temática nas bases de dados, bem como formar grupo de pesquisa com a mesma linha de pesquisa.

No contato com o orientador, dificuldades no instrumento de estudo e ABNT (H10).

Falta de artigos relacionados ao tema, não conhecer metodologia científica e principalmente a ausência de orientador (H27).

Formar grupo que aceite realizar pesquisa com o mesmo tema e orientador (G09).

\section{DISCUSSÃO}

Os dados do presente estudo mostram a necessidade de incentivo à pesquisa durante a graduação, preferencialmente sendo abordada desde os primeiros semestres do graduando, estimulando o saber, a importância e a realização desta atividade. Tão logo, destaca-se a importância em obter o conhecimento exposto sobre metodologia científica, despertando a motivação para realizar pesquisa, desenvolvendo o pensamento crítico do aluno e proporcionando contribuição para ciência (ARAÚJJO AML, et al., 2015).

A abordagem quantitativa evidenciou uma população jovem feminina no curso de graduação de Enfermagem, exibindo similaridade a estudos prévios no mesmo tipo de amostra (XIMENES NETO FRG, et al., 2017; AYOAMA EA, et al., 2018). Historicamente, a enfermagem vem se mostrando como uma profissão culturalmente e majoritariamente feminina, no entanto, esse perfil vem mudando ao decorrer dos anos, observando-se uma crescente atuação do gênero masculino (MACHADO MH, et al., 2016).

Identificou-se que maioria dos acadêmicos deste estudo cursava o turno matutino, divergindo de outro estudo que mostrou maior prevalência noturna (AYOAMA EA, et al., 2018). Hirsch CD, et al. (2018) afirma que quando há a co-associação de trabalho e estudo, os acadêmicos tendem a optar por estudar no noturno, essa rotina intensa pode repercutir negativamente no rendimento acadêmico, cansaço físico e psicológico 
dos mesmos, caracterizando-se como potencial gatilho de estresse. Considerando-se o ritmo acelerado de inovação biotecnológica, a formação do enfermeiro deve atender as necessidades do mercado, cuja exigência é cada vez maior por profissionais com múltiplas funcionalidades e competências, com prática clínica baseada em evidência e habilidade/aprendizado interdisciplinar na pesquisa clínica e translacional (SLATTERY MJ, et al., 2016). Dessa forma, a experiência de pesquisa deve ser precoce na graduação sendo essencial para fomentar e suprir a essa demanda exigida (MORALES DX, et al., 2017; ARAÚJO AML, et al., 2015).

Neste estudo, a vivência da pesquisa pelo acadêmico de enfermagem foi limitada na graduação de forma significativa, isso pode ser observado pela baixa produção científica indexada como artigos em revistas cientificas ou capítulos de livros. Publicações sobre a importância da produção compartilhada entre docente e discente têm sido negligenciadas na literatura (MORALES DX, et al., 2017). Apenas um estudo exibiu resultados semelhantes em relação à tímida publicação cientifica, ressaltando-se a subrepresentação da pesquisa na vivência acadêmica (ARAÚJO AML, et al.,2015).

Os acadêmicos de enfermagem abordados afirmaram ter recebido incentivo à pesquisa das instituições privadas e docentes, no entanto, a maioria apenas apresentou produção de resumos destinados à eventos científicos apenas. Araújo AML, et al. (2015) afirma que este tipo de produção demanda pouco tempo de dedicação do binômio orientador-orientando em sua criação e execução, limitando-se a vivência na pesquisa pelo acadêmico. Morales DX, et al. (2017) acrescenta que mais de um ano de vivência na pesquisa pelo acadêmico é necessária para aprimorar habilidades e gerar produtividades.

Outros estudos brasileiros enumeram alguns obstáculos associados a produção cientifica reduzida como ausência de carga horária destinada aos docentes às pesquisas, de incentivo financeiro, questões éticasburocráticas, bem como plágio, fraude e publicações duplicadas, o que limitam as oportunidades de iniciação científica (ARAÚJO AML, et al., 2015; BOZZO A, et al., 2017; AL-GHAREEB A, et al., 2018; BARATA RB, 2019). Assim, a vivência limitada à pesquisa durante a graduação resulta em inexperiência ou pouca habilidade no processo de pesquisa cientifica, o que repercute na escolha e execução tardia do TCC. Um único estudo demonstrou similaridade aos nossos resultados, embora tal prática seja comum entre os graduandos (SILVA MMS, 2016).

Sabe-se que o docente é a peça chave no despertar da pesquisa científica na graduação, pois ele ensina habilidades de pesquisas sistematicamente integradas à grade curricular de enfermagem e a escrita do TCC é uma estratégia potencial para a consolidação delas (NORDSTEIEN A, et al., 2017; CAMARGO FC, et al., 2018).

O docente é capaz de influenciar na escolha da temática do TCC mediante a sua atuação profissional, contudo, as temáticas fora da linha de pesquisa do docente são recusadas pelo mesmo (ALMEIDA LS, et al., 2018). Neste estudo, os temas escolhidos pelos acadêmicos para o TCC também foram relacionados à área de atuação do docente e a satisfação do acadêmico foi evidente, semelhante ao resultado de um estudo brasileiro (GEWEHR D, et al., 2016).

Interessantemente, a expectativa de atuação profissional do acadêmico também pode ser motivada pelo docente, isso foi destacado nesse estudo. Predominantemente, os acadêmicos manifestaram maior afinidade pela modalidade assistencial e por último a pesquisa. Resultado similar foi identificado em um estudo do nordeste brasileiro, em que 54\% relatou preferência pela assistência como segmento profissional (LIMA RBS, et al., 2018). Tal achado revela a dificuldade de libertação do paradigma histórico da profissão de potencial cunho assistencial e um distanciamento da pesquisa, embora a transferibilidade pesquisa-assistência seja mútua e interdependente (SCOCHI CGS, et al., 2015; SILVA ÍR, et al., 2019). Lima RBS et al. (2018) sintetiza que os profissionais não podem estar focados apenas no cuidar em si e sim nas circunstâncias que possam levar ao aperfeiçoamento desse cuidado pela pesquisa.

$\mathrm{Na}$ abordagem qualitativa do presente estudo, avaliou-se a percepção dos acadêmicos de enfermagem quanto a três categorias (i) a relevância da pesquisa científica na graduação, (ii) a metodologia científica enquanto disciplina e (iii) as dificuldades enfrentadas pelos acadêmicos na realização do TCC. A percepção dos acadêmicos de enfermagem sobre a relevância da pesquisa científica durante a graduação foi 
reconhecida, ressaltou-se que a mesma é uma ferramenta capaz de desenvolver habilidades assistenciais, pensamento crítico, bem como a melhoria do currículo lattes.

Ayoama EA, et al. (2018) ratifica como fundamental à formação acadêmica e profissional, capaz de desenvolver habilidades, pensamento crítico, aperfeiçoamento da prática baseada em evidências, resultados coincidentes com o presente estudo. O uso da pesquisa é essencial à prática baseada em evidência e precisa ser aplicada desde a graduação (NORDSTEIEN A, et al., 2017). O estudo de Araújo AML, et al. (2015) reforçou as opiniões positivas sobre a importância da pesquisa na graduação, em que $37,1 \%$ de acadêmicos relataram que a atividade científica incentiva o desenvolvimento profissional e que os grupos de pesquisa têm contribuído para tal. Acredita-se que a exposição dos acadêmicos de enfermagem a experiências de pesquisa cientifica mais intensas e realistas é responsável por despertar maior interesse e desenvolvimento de habilidades na área (SLATTERY, et al., 2016). Embora a disciplina metodologia científica seja uma ferramenta primordial à pesquisa, os resultados deste estudo revelaram insatisfação significativa, com relatos de distanciamento e falta de afinidade pelos acadêmicos.

O estudo de Oliveira TAB e Valença KFP (2015) também destacou a aversão dos acadêmicos à disciplina, por requerer regras rigorosas à escrita e formatação da escrita científica, bem como as dificuldades experimentadas com o dinamismo das regras da ABNT que se atualizam constantemente. Adicionalmente, 0 tipo de abordagem tradicionalmente teórica da disciplina, com pouca aplicabilidade em sala de aula tem desfecho negativo sobre a afinidade e dificuldade de escrita científica do acadêmico de enfermagem (ARAÚJO AML, et al., 2015). Embora a relevância e complexidade da metodologia científica associada as diretrizes preconizadas pela Resolução oㅜ 573/2018 estejam legitimadas, o Ministério da educação (MEC) ampliou a carga horária de aulas à distância (EAD) em $40 \%$ dos cursos presenciais (Portaria № 1.428/2018), o que permitiu a inclusão da disciplina metodologia científica nesta modalidade (MINISTÉRIO DA EDUCAÇÃO-BRASIL, 2018). Esta forma de abordagem prejudicou o ensinamento da disciplina conforme destacado nas falas dos acadêmicos de uma instituição de ensino deste estudo. Alternativamente, a afinidade pela disciplina foi correlacionada ao professor que a ministrou presencialmente na outra IES (MORALES DX, et al., 2017).

É válido ressaltar que a Resolução CNE/CES № 3/2001 não detalha o tipo ou período de abordagem da disciplina, permitindo que ela seja executada de forma presencial ou a distância de acordo com a adequabilidade da IES (MINISTÉRIO DA EDUCAÇÃO, BR, 2001). Não foram encontradas publicações referentes ao assunto, mas outras disciplinas quando ministradas a distância mostram resultados conflituosos, relacionados a satisfação pela acessibilidade e flexibilidade de tempo e insatisfação deflagrada pela limitação de conhecimento, falta de domínio da plataforma educacional, de esclarecimento de dúvidas, de motivação e de cobranças vivenciadas na modalidade presencial (PISSAIA LF, et al., 2017).

Com isso, uma melhor abordagem da disciplina e sua aplicabilidade são imperiosas ao desenvolvimento de habilidades para a produção cientifica (ASSOCIAÇÃO BRASILEIRA DE NORMAS TÉCNICAS, 2019). As maiores dificuldades vivenciadas durante o TCC estão relacionadas aos métodos científicos, as bases de dados e as dinâmicas exigências estabelecidas pela Associação Brasileira de Normas Técnicas (ABNT), que são habilidades abordadas pela disciplina e vivenciadas na pesquisa (RODRIGUES FWA e RAMOS ABB, 2019). No Brasil, as linhas de pesquisas sobre metodologia científica são escassas em relação ao exterior, reforçando a necessidade de publicações, bem como o desenvolvimento de produções que abordem 0 binômio orientador-orientando, uma vez que isso repercute na qualidade de ensino e aprendizagem, bem como a aplicabilidade durante a pesquisa (NÓBREGA MH, 2018; JÚNIOR ER, et al., 2018; CLEMENTE FAZ e SANTOS LCB, 2015).

Para que se garanta a formação de jovens cientistas é preciso viabilizar acesso a programas de iniciação cientifica por parte das instituições de ensino, enquanto dos alunos é necessária dedicação reponsabilidade ética e iniciativa para vencer dificuldades. Nesse sentido a importância do incentivo a divulgação cientifica no Brasil, aliado ao investimento financeiro ocorre avanços na ciência, pesquisa, levando ao desenvolvimento de tecnologia e inovação que são primordiais para o desenvolvimento da nação (LIMA MO e VIANA GMR, 2017). 


\section{CONCLUSÃO}

Primeiramente, este estudo destaca-se pela importância da temática ainda pouco abordada. Em segundo lugar, ele mostra que há reconhecimento da importância da pesquisa científica pelos acadêmicos embora haja dificuldades e limitações da sua vivência devido a experiências tardias com o TCC. Em terceiro lugar, infere-se que as diretrizes curriculares são promissoras, mas para sua aplicabilidade é necessário que ocorra reestruturação multidimensional relacionada as entidades de classe, às instituições de ensino superior e agências de fomento a fim de permitir que docentes tenham melhores condições de formar enfermeiros cientistas. As limitações deste estudo incluíram a percepção unicamente sob olhar dos acadêmicos não levando em consideração a opinião dos docentes e dos responsáveis pela instituição. Ademais, as questões burocráticas de aprovação extrapolaram o tempo planejado para a coleta de dados em mais duas IES, o que reduziu o $\mathrm{n}$ amostral. Sugere-se que estudos adicionais devam ser desenvolvidos a fim de melhorar os métodos de ensino-aprendizagem e sua aplicabilidade na pesquisa durante a formação deste profissional.

\section{REFERÊNCIAS}

1. ABRAMSON JH. WINPEPI updated: Computer programs for epidemiologists, and their teaching potential. Epidemiologic Perspectives \& Innovations. 2011; 8(1): 2-9.

2. AL-GHAREEB $A$, et al. Retraction of publications in nursing and midwifery research: $A$ systematic review. Int $J$ Nurs Stud, 2018; 81:8-13.

3. ARAÚJO AML, et al. A pesquisa científica na graduação em enfermagem e sua importância na formação profissional. Revista de enfermagem UFPE on line, 2015; 9(9): 9180-7.

4. ASSOCIAÇÃO BRASILEIRA DE NORMAS TÉCNICAS. Normalização, elaboração e participação. Disponível em: http://www.abnt.org.br/12-normalizacao/24-normalizacao-brasileira. Acesso em: 08 de jun. 2019.

5. AYOAMA EA, et al. Perfil socioeconômico e cultural dos discentes de enfermagem das faculdades integradas da União Educacional do Planalto Central (FACIPLAC). REFACI, 2018; 2(2): 1-12.

6. BARATA RB. Desafios da editoração de revistas científicas brasileiras da área da saúde. Ciência \& Saúde Coletiva, 2019; 24(3): 929-939.

7. BARDIN L. Análise de conteúdo. 70 ed. Reto LA, Pinheiro A, translator. São Paulo, 2011;65p.

8. BOZZO A, et al. Retractions in câncer research: a systematic survey. Res Integr Peer Rev, 2017; 2(1): 5.

9. BRASIL. Ministério da Educação. Gabinete do Ministro. Portaria no 1.428, de 28 de dezembro de 2018. Dispões sobre a oferta, por Instituições de Educação Superior- IES, de disciplina na modalidade a distância em cursos de graduação presencial. Diário Oficial da União, Brasília, DF,2018.

10. CAMARGO FC, et al. Competences and Barriers for the Evidence-Based Practice in Nursing: An Integrative Review. Rev Bras Enferm, 2018;71(4):2030-8.

11. CLEMENTE FAZ, SANTOS LCB. Desmistificando o Trabalho de Conclusão de Curso (TCC) da graduação. Revista educação, 2015; 10(2): 23-39.

12. ALMEIDA LS, COQUEIRO JM, FIGUEIREDO TAM. A formação do enfermeiro sob olhar do estudante: linhas fortes e fracas. Revista Uningá, 2018;55(3): 183-198.

13. GEWEHR D, et al. Metodologias ativas de ensino e de aprendizagem: uma abordagem de iniciação a pesquisa. Revista Multidisciplinar de Licenciatura e formação docente Ensino \& Pesquisa, 2016;14(1):225-246.

14. HIRSCH CD, et al. Fatores percebidos pelos acadêmicos de enfermagem como desencadeadores do estresse no ambiente formativo. Rev. Texto Contexto Enferm, 2018;27(1):1-11.

15. JÚNIOR ER, et al. Relações interpessoais e sua influência na satisfação dos acadêmicos. Revista de Gestão e Secretariado, 2018; 9(3):206-228.

16. LIMA MO, VIANNA GMR. Divulgação científica: reponsabilidade e importância. Revista Pan-Amazônica de Saúde, 2017; 8 (4) :7-8.

17. LIMA RBS, et al. Motivos para escolha da profissão de enfermeiro. Revista Baiana de enfermagem, 2018; 32 : 1-9.

18. MACHADO MH, et al. Características gerais da enfermagem: o perfil sócio demográfico. Enferm. Foco, 2016;7(Esp):09-14.

19. MINISTÉRIO DA EDUCAÇÃO (BR). Conselho Nacional de Educação. Resolução do CNE/CES no 3 de 7 de novembro de 2001. Institui as Diretrizes Curriculares Nacionais do Curso de Graduação em Enfermagem.

20. MORAES A, et al. The nurse training in research in the undergraduate education: teaching perceptions. Rev Bras Enferm, 2018;71(Suppl 4):1556-63.

21. MORALES DX, et al. Increasing research productivity in undergraduate research experiences: Exploring predictors of collaborative faculty-student publications. CBE-Life Sciences Education, 2017; 16 (3): ar42. 
22. XIMENES NETO FRG, et al. Perfil sociodemografico dos estudantes de enfermagem da Universidade Estadual Vale do Acarau (UVA). Enferm Foco, 2017;8(3):75-79.

23. NÓBREGA MH. Orientandos e Orientadores no Século XXI: desafios da pós-graduação. Educação \& Realidade, 2018;43(3):1055-1076.

24. NORDSTEIEN A, HORNTVEDT MT, SYSE J. Use of research in undergraduate nursing students' theses: A mixed methods study. Nurse education today, 2017; 56: 23-28.

25. OLIVEIRA TAB, VALENÇA KFP. A importância da metodologia científica para o ensino e aprendizagem no ensino superior. Educere. In: XII Congresso Nacional de Educação, 2015: 7480-7470. Disponível em: https://educere.bruc.com.br/arquivo/pdf2015/17807_10482.pdf. Acesso em: 17 de out. 2019.

26. PISSAIA LF, et al. Percepções de enfermeiros quanto à realização de cursos de pós-graduação na modalidade de ensino a distância (EAD). Revista Signos, 2017;38(2): 63-737.

27. PRAÇA FSG. Metodologia da pesquisa científica: organização estrutural e os desafios para redigir o trabalho de conclusão. Revista eletrônica Diálogos Acadêmicos, 2015;8(1):72-87.

28. RODRIGUES FWA, RAMOS ABB. Metodologia científica: análise e reflexão sobre a percepção dos graduandos. International Journal Education and Teaching (PDVL), 2019; 2(1): 47-60.

29. SCOCHI CGS, et al. Nursing Doctorates in Brazil: research formation and theses production. Revista latino-americana de enfermagem, 2015; 23(3): 387-394.

30. SILVA ÍR, et al. Aprender pela pesquisa: do ensino da ciência ao campo assistencial da enfermagem. Escola Anna Nery Revista de Enfermagem, 2017; 21(4):1-8.

31. SILVA ÍR, et al. Enfermeiro pesquisador e enfermeiro assistencial: construção e projeção de identidades polimorfas. Rev. Bras. Enferm, 2019;72(Suppl 1): 204-212.

32. SILVA MMS. A importância do conhecimento científico na universidade e sua contribuição para a semana científica da faculdade R.Sá. X SEMANA DE INICIAÇÃO CIENTÍFICA AS FACULDADE R. SÁ, 2016, Picos, PI. Disponível em: https://www.faculdadersa.edu.br/artigos-2017-gt-06. Acesso em: 17 de out. 2019.

33. SLATTERY MJ, et al. An undergraduate research fellowship program to prepare nursing students for future workforce roles. Journal of Professional Nursing, 2016; 32(6): 412-420. 